\title{
THE EFFECT OF ANGLES OF THE BUILDING CORNERS ON ITS PATTERN OF EXPLOSIVE LOADING OF SIDE FACES IN THE CASE OF THE EXTERNAL EXPLOSION
}

\author{
Alireza Haji Ghorbani Zadeh ${ }^{1 *}$, Mostafa Amini Mazraeno ${ }^{2}$, Amin Moslemi Petrudi ${ }^{3}$ \\ 1,2 Department of Civil Engineering, Imam Hussein University, Tehran, Iran \\ ${ }^{3}$ Department of Mechanical Engineering, Tehran University, Iran \\ *Email: \{Ali.rhgz@Gmail.com\}
}

\begin{abstract}
In this paper, guidelines such as UFC-3-340-02 have been developed to design structures against explosive loads. In UFC regulations, a number of simplistic assumptions are considered in order to calculate the explosive loading pattern of building constructions. If one of these assumptions is violated, an explosive loading pattern of building materials should be obtained using laboratory and numerical methods. The change in the corners of the building (non-impermeable corners of the building cone) violates one of the assumptions of the UFC Code. In this study, we simulate explosive phenomena in the AUTODYN hydro code to calculate the explosive loading pattern of the side faces of the building. The effect of four variables including the left and right coronal angles from the front, the forward aspect ratio and the explosive space distance from the building on the side faces impact explosion pattern using 25 Taguchi methods suggested. As a result of this study, the opening of the cornea from the front directly affects the maximum and impulse pressure on lateral faces and the speed of the wave transition.
\end{abstract}

Keywords: Explosion, The corners of the building, Numerical simulation, Taguchi method.

\section{Introduction}

In everyday life, explosions have been a significant and always alarming topic for human beings. The explosion can occur deliberately in the context of war and terrorist operations, or it can occur as a result of a careless or sudden environmental readiness, such as a gas capsule explosion [1]. Most structures are highly vulnerable to explosive threats, because the loading under which the structure is designed is often less than the force applied to the structure by blasting, and additional loading is required to design the structures against the blast load. Apply to the structure and analyze the structure under its influence. Given the random nature of blast loading and other uncertainties, it is logical that in order to determine the vulnerability of structures to this loading, researchers with laboratory and field studies provide solutions and methods in various formats to facilitate the analysis and design of structures under this loading. The geometry, shape, and cross-section of buildings directly influence their resistance to the blast wave front. Changes in the angles of the building (changing the building section) can change the speed at which the blast wave front passes through the building surface. By decreasing the fracture rate of the structure in front of the blast wave front, the velocity and wave motion (faster release) are increased and the duration of impact on the structural facets also decreases [2].

\section{LITERATURE REVIEW}

UFC 334002 was issued by the US Department of Defense in 2002 after conducting numerous tests and numerical simulations in the field of loading, analysis and design of explosion-proof structures. In 2014, a newer version of the Code of Conduct was introduced, which has a more attractive appearance and better and more useful diagrams [3]. In the second chapter of this regulation by explosion loading pattern, under the influence of various variables such as type, weight and geometry of explosive material, location of explosion (explosion 
in open air, explosion in air and surface explosion), distance of explosive to structure, explosion Internally and externally, the geometry of the explosion-proof structure and several other variables that were able to be investigated and presented simplified diagrams for the purpose of comprehensiveness of the UFC rules are provided. Although a lot of experimentation has been done with a variety of variables to compile this code, the UFC rules put some restrictions on their diagrams and tables because of the explosive loads that are once irregularly dynamic and simplify diagrams and tables. While these limitations have helped to have simple, functional diagrams and relationships, but on the other hand, the problem is difficult to solve through numerical simulations and new experiments if the problem is in a way that cannot be solved using UFC diagrams and diagrams. The issue went on to point out some of the issues in some explosive loading issues. An article titled " Angle of incidence effects on far-field positive and negative phase blast parameters" was published in 2015 by Sam et al. The results of this paper are the result of a series of highprecision experiments, which used pressure transducers to store time pressure history on a large screen. The rigid object with variable angles for variable dimensions of PE4 hemispherical spend at constant distances has been studied. Interaction results show that the overpressure, impulse, and wave arrival time are highly dependent on the target angle for the positive phase, and are not dependent on the target angle in the negative phase [4]." Explosion protection-architectural design, urban planning and landscape planning" is a 2009 article by Gebbeken et el. The reflected time pressure diagram, which is the design load, depends on the geometry and shape of the structure that reflects the explosion wave. This article presents the influence of geometry or shape of the structure on the explosion design load for a number of shapes. The shapes investigated include horizontal shapes (such as part of closed columns), vertical shapes (such as views), and specific shapes of buildings (spatial structures). According to the results of this paper, by reducing the amount of fractures at the surface and cross-section of the building, the blast wave front moves faster on the surface and decreases the duration of its impact on the surface, which reduces the unit impulse value. Reducing the amount of brittleness can be caused by the use of circular sections, or by increasing the angles and brittle angles of the building, meaning that the geometric shape of the building is closer to the circle. As a result, the shape of a structural or building member can drastically reduce the design load [2]. Numerous articles have been published in the field of numerical simulation of explosive phenomena in shown table 1 .

Table 1

Literature review of blast wave.

\begin{tabular}{|c|c|c|}
\hline Author(s) & Parameters & Year(s) \\
\hline $\begin{array}{l}\text { Ling et al } \\
\quad[15]\end{array}$ & $\begin{array}{l}\text { Simulation and scaling analysis of a } \\
\text { spherical particle-laden blast wave }\end{array}$ & 2018 \\
\hline $\begin{array}{l}\text { Kim et al } \\
{[16]}\end{array}$ & $\begin{array}{l}\text { Investigation of blast wave effects } \\
\text { on containment wall and steam } \\
\text { generator }\end{array}$ & 2018 \\
\hline
\end{tabular}

Metzger et al Fast radio bursts as synchrotron

[8] maser emission from decelerating 2019 relativistic blast waves

\begin{tabular}{|c|c|c|}
\hline $\begin{array}{c}\text { Rashad et al } \\
{[7]}\end{array}$ & $\begin{array}{c}\text { Experimental and numerical } \\
\text { investigation of RC sandwich } \\
\text { panels with helical springs under } \\
\text { free air blast loads }\end{array}$ & 2019 \\
\hline Yang [6] & $\begin{array}{l}\text { Anti-explosion Performance of } \\
\text { Engineered Cementitious } \\
\text { Composite Explosion-Proof Wall }\end{array}$ & 2020 \\
\hline $\begin{array}{l}\text { Wang et al } \\
{[5]}\end{array}$ & $\begin{array}{l}\text { state-of-the-art review on blast } \\
\text { resistance and protection of high } \\
\text { dams to blast loads }\end{array}$ & 2020 \\
\hline
\end{tabular}

Ivančo et al Different Approaches of Numerical [17] Simulation of Blast

2020

\section{EXPLOSION DETECTION}

Explosion is a phenomenon of a random nature that affects structures or obstacles close to or in contact with the environment due to the shock wave and sudden perturbation in its surroundings. An explosion occurs when a large amount of energy is released quickly and suddenly in the form of heat and pressure. Following the explosion, a shock wave is created around the environment. The shock wave is a nonlinear wave family of longitudinal mechanical 
waves that pressurize the environment [9]. The stages of formation and advancement of the blast wave front include severe and sudden release of energy, initial crack propagation, radial blast propagation, impedance collision, secondary crack propagation, amplified wave reflection, peak pressure reduction at ambient pressure. The pressure drop is lower than the ambient pressure before the explosion and the pressure returns to ambient pressure [10]. When a body is exposed to the blast wave front, its procedural pressure rises and reaches its maximum in a very short moment. This pressure simply surrounds and surrounds the body around it, which can increase stresses and deformities and have a direct impact on its function [11].

\section{Explosion outside the structure}

The general form of the pressure history of the shock wave time of an open-air blast is shown in Figure 1. The wave front is necessarily vertical due to the sudden increase in pressure caused by the explosion. The maximum pressure caused by the $\mathrm{P}_{\mathrm{so}}$ explosion is at the end of this initial phase (rise time). The pressure caused by the explosion is the pressure applied to the surface parallel to the direction of propagation. The emission velocity $\mathrm{V}$ decreases with time and distance, but is usually greater than the speed of sound in the environment. The shock front reaches the target at $T_{a}$. After $T_{r}$ seconds after reaching the target $\left(\mathrm{T}_{\mathrm{a}}\right)$, the pressure will reach its maximum value, $\mathrm{P}_{\text {so }}$. Since the time interval between the shock front reaching the target and the maximum pressure occurring is very short, $T_{r}$ is assumed to reach the maximum pressure value immediately after the shock front arrives. The maximum pressure $\mathrm{P}_{\mathrm{so}}$ decreases during $T_{\mathrm{o}}$ and is equal to the initial ambient pressure, which is defined as the positive phase of the pressure blow. After this phase, the negative phase occurs, which will continue for $T_{o}^{-}$ during which time the pressure will be lower than the initial ambient pressure and the wind direction (particle motion) reversed. The negative phase of the design is of little importance and is usually overlooked. The value of the impact caused by the blast wave is equal to the area under the positive phase curve of the time pressure curve (impulse) and is expressed by $i_{\mathrm{s}}$.

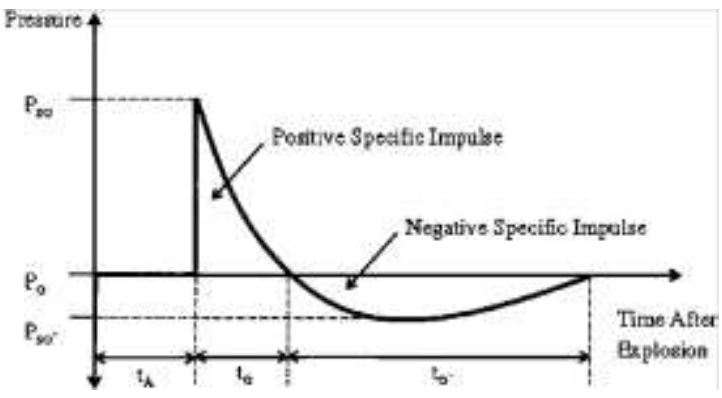

Fig.1. Curve of time-pressure diagram of the explosion [18].

The shock wave will be propagated as described above, as long as there is no obstacle to it. If the shock wave reaches a surface that is not parallel to the propagation (such as a wall or a structure), reflective pressure will be generated. The reflected pressure has the same general form of pressure produced, but as shown in Figure 2, its maximum pressure value is greater than the maximum pressure of the initial wave. The reflection pressure depends on the initial wave, the angle of inclination between the reflecting surface and the direction of the reflection.

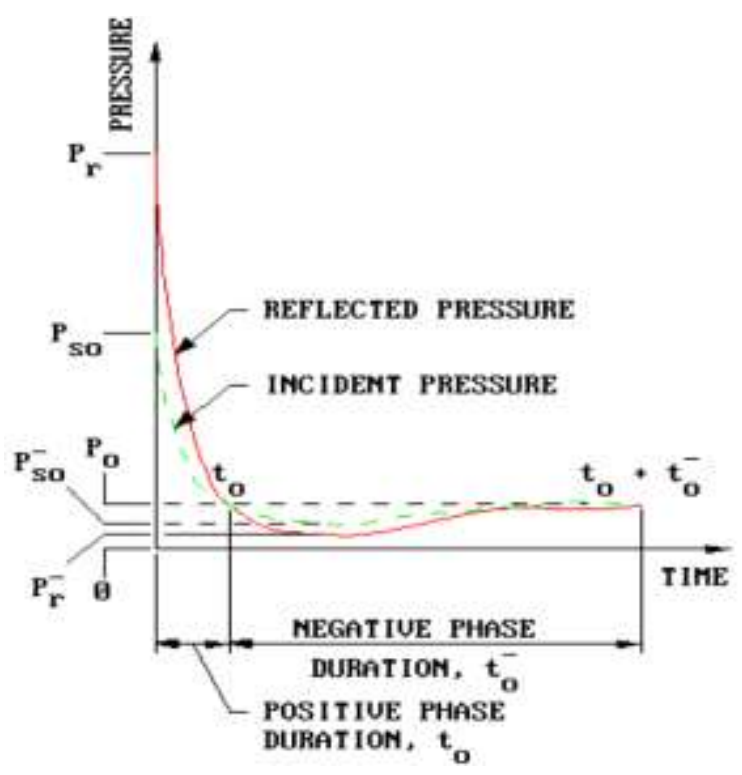

Fig. 2. Reflective wave time pressure diagram [19].

The survival time of the reflection pressure depends on the size of the reflecting surface that determines the flow rate around the surface. This secondary 
current causes the pressure wave pressure reflected throughout the temperature pressure history range, from the maximum pressure region to the minimum pressure region, and to the static pressure, that is, the amount of pressure produced at equilibrium with the generated high velocity. Is, arrives. If the secondary current does not cause the reflection pressure to deteriorate (such as in the case where the infinite plane wave contacts the infinitely long wall), the initial generated wave at all points of the reflected surface and the duration of the reflected wave (positive phase) equal to the duration The durability of the initial generated wave will be. The single impulse for a fully reflected wave in the positive phase range is indicated by $i_{n}$. The positive phase wavelength $L_{w}$ is equal to the distance between the blast point and the last point that experiences positive pressure at a given time [12].

\section{Sensitivity analysis of numerical results}

Hydro codes are one of the most widely used methods for analysing, exploding, and blasting blast analysis. AUTODYN software is an integrated hydro code that can be used to solve a wide range of problems like wave, explosion, penetration, collision and etc. In order to evaluate the accuracy of the numerical simulation results in AUTODYN software a quadrilateral building with upright sidewalls is subjected to a wave of explosion of a TNT rectangular cube. Then the results of numerical simulation with the proposed values of UFC 3340 02 are investigated.

Table 2

Characteristics of constant parameters.

\begin{tabular}{lcc}
\hline $\begin{array}{l}\text { Distance of explosive } \\
\text { material from structure }\end{array}$ & $65.62 \mathrm{ft}$ & $20 \mathrm{~m}$ \\
\hline Scaled distance & $5.39 \mathrm{ft} / \mathrm{lb}^{1 / 3}$ & $2.14 \mathrm{~m} / \mathrm{kg}^{1 / 3}$ \\
\hline $\begin{array}{l}\text { Explosive material } \\
\text { Weight (TNT) }\end{array}$ & $1796.77 \mathrm{lb}$ & $815 \mathrm{~kg}$ \\
\hline $\begin{array}{l}\text { Dimensions of } \\
\text { explosive material } \\
\text { rectangular cube }\end{array}$ & $\begin{array}{l}3.28 \times 3.28 \\
\times 1.64 \mathrm{ft}\end{array}$ & $\begin{array}{l}100 \times 100 \\
\times 50 \mathrm{~cm}\end{array}$ \\
\hline $\begin{array}{l}\text { The length of the front } \\
\text { of the structure }\end{array}$ & $19.69 \mathrm{ft}$ & $6 \mathrm{~m}$ \\
\hline Side length of structures & $13.12 \mathrm{ft}$ & $4 \mathrm{~m}$ \\
\hline Structural height & $9.84 \mathrm{ft}$ & $3 \mathrm{~m}$ \\
\hline
\end{tabular}

The explosion phenomenon is a type of surface explosion. The explosive is of TNT type and is considered as a rectangular cube. The numerical solver of the explosive zone and the forward of the blast wave front in the air is of the Eulerian type and has a grid size of $100 \mathrm{~mm}$. By default, AUTODYN software provides extra pressure for the air environment. By modifying the overpressure and setting it to zero, the effect of overpressure on the simulated model will be neutralized and the simulated problem characteristics will be matched to the actual model. The boundary condition considered around the Eulerian environment (the explosion wave propagation environment) is the outflow of all aspects except the surface. The structure is $35 \mathrm{MPa}$ with a $200 \mathrm{~mm}$ Lagrangian solver which is rigidly modelled with fixed boundary condition and without any displacement. In fact, by considering this boundary condition and closing the deformations and shifts of the building facets in all directions, conditions are being prepared for examining merely the pattern of explosive loading on the building sides.

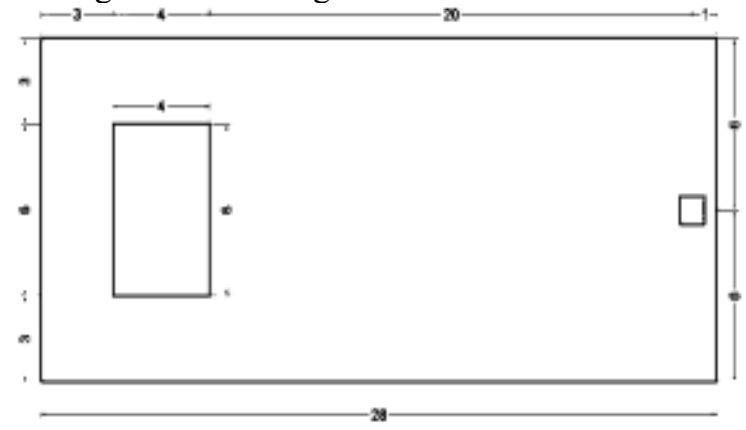

Fig. 3. Simulated model plan in AUTODYN software.

In the UFC regulations, the proposed Time-Pressure diagram for each side seems to be the average TimePressure diagram of the explosion wave on that side. In numerical simulation in AUTODYN software, the barometers defined on different sides provide only Time-Pressure diagrams at the same point, while having to obtain the average pressure value on the side (total Time-Pressure diagram), and to do so according to The coordinates and order are defined on the whole surface of each face, using the mean time pressure diagram of the face. By having TimePressure diagrams at the position of the barometers defined on the face, it is obtained by relation of (1) the average Time-Pressure diagram of the whole face. 


$$
\begin{aligned}
\overline{P(t)} & =\frac{\iint P(t \cdot x \cdot y) \cdot \emptyset(x \cdot y) d x d y}{\iint \varnothing(x \cdot y) d x d y} \\
\overline{P(t)} & =\frac{\sum P\left(t \cdot x_{i} \cdot y_{i}\right) \cdot \emptyset\left(x_{i} \cdot y_{i}\right) \cdot \Delta x_{i} \cdot \Delta y_{i}}{\sum \emptyset\left(x_{i} \cdot y_{i}\right) \cdot \Delta x_{i} \cdot \Delta y_{i}}
\end{aligned}
$$

In order to simplify how to obtain the mean TimePressure diagram, relation (2) can be used instead of relation (1). The components that affect the mean time diagram of each aspect include the following 3 components:

1. $\left(t \cdot x_{i} \cdot y_{i}\right)$ : Time-Pressure diagram of each of the defined pressure gauges on the face.

2. $\emptyset\left(x_{i}, y_{i}\right)$ : The deformation function of each of the defined pressure gauges on the face, in two directions $\mathrm{x}$ and $\mathrm{y}$, which can be calculated by two methods. In the first method, using the relations (3) and (4), two components $\varnothing\left(x_{i}\right)$ and $\varnothing\left(y_{i}\right)$ are obtained, and then using the relation (5), the function of corner change in the position of each barometer is obtained (analytical method).

$$
\begin{aligned}
& \emptyset\left(x_{i}\right)=\frac{16 x_{i}^{2}\left(l_{x}-x_{i}\right)^{2}}{l_{x}^{4}} \\
& \varnothing\left(y_{i}\right)=\frac{16 y_{i}^{2}\left(l_{y}-y_{i}\right)^{2}}{l_{y}^{4}} \\
& \varnothing\left(x_{i}, y_{i}\right)=\varnothing\left(x_{i}\right) . \emptyset\left(y_{i}\right)
\end{aligned}
$$

In the second method, based on the static analysis of the building in exchange for applying uniform pressure on the target unit, by obtaining the rate of change of location of each barometer in proportion to the maximum displacement on the surface (maximum pressure barometer), the value of the deformation function or $\varnothing\left(x_{i}, y_{i}\right)$ is calculated in the position of each barometer (numerical method).

3. $\Delta x_{i} . \Delta y_{i}$ : Area covered by each of the barometers. Therefore, the mean Time-Pressure diagram of each face is obtained from both analytical and numerical methods [13].

The numerical simulation of the explosive phenomenon with the above specifications was performed in AUTODYN software and we obtained the Time-Pressure diagrams of the side faces and roof from both analytical and numerical methods. Then, the proposed Time-Pressure diagram of the UFC code is obtained for the side faces and roof and compared with the numbers obtained from the numerical simulation in AUTODYN. As the blast wave progresses and moves on the side faces of the structure, after reaching the sides and the roof, the amount of pressure decreases and also the duration of the positive phase continues. Figure 4 shows the average Time-Pressure diagram obtained from numerical simulation for the left and right sides of the building as well as the roof of the building (obtained from both analytical and numerical methods) along with the Time-Pressure diagram of the UFC regulations. The main components of these diagrams are compared in Table 2, which shows that the percentage of error between their results is permissible and can be acceptable. It is also shown in Figure 4 and Table 2 that the median TimePressure diagrams obtained are consistent with both analytical and numerical methods. As a result, both analytical and numerical methods can be used to calculate the mean time side faces and roof pressure diagrams of the building. Medium Time-Pressure diagrams of each corner of buildings in this study are obtained by analytical method.

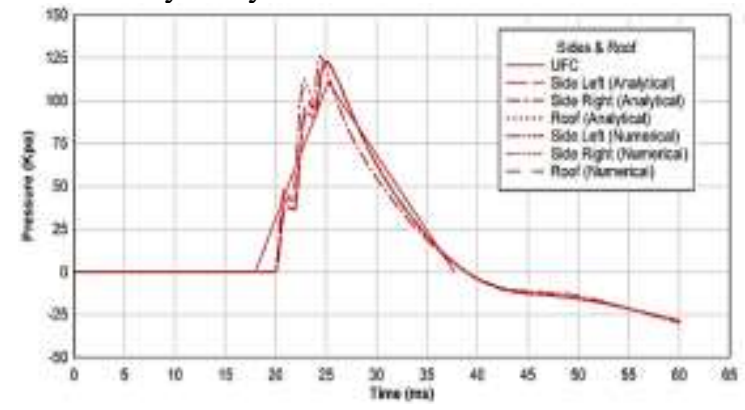

Fig. 4. Time-Pressure diagram of the side faces and roof of the building.

\section{Statement of problem}

One of the simplifying conditions in the UFC regulations is that the corners of the building are upright. In this study, assuming that the condition of the right angles of the angle is violated, an attempt is made to study its effect on the loading pattern of the side face and roof of the building by numerical simulation in the AUTODYN hydraulic code. In this study, the effect of four variables including the angle of the left corner of the front face $\left(\alpha_{\text {left }}\right)$, the angle of the right corner of the front face $\left(\alpha_{\text {right }}\right)$, the length of the front face $(\mathrm{L})$ and the distance of the explosive from the building (R) on the explosive loading pattern The side face and roof of the building are investigated. The variables of the length of the front face and the distance of the explosive from the building are variables that can only be investigated 
by manual calculations. But here they are considered along with two other variables, and by examining the results of numerical simulation, their effectiveness is investigated in cases where the angles of the angle also change. The explosion phenomenon is a type of surface explosion. The TNT explosive weighs 815 $\mathrm{kg}(1796.77 \mathrm{lb})$ and is considered to be a rectangular cube measuring $100 \times 100 \times 50 \mathrm{~cm}$. The numerical solver of the blast area and the blast wave advance zone is $100 \mathrm{~mm}$ in size and in the air environment, and the excess air pressure is set to zero. The boundary condition considered around the Euler environment (Explosive Wave Advanced Environment) is the flow out the boundary in all faces except the ground level. The structure is 35 $\mathrm{MPa}$ with a $200 \mathrm{~mm}$ Lagrangian solver which is rigidly modelled with fixed boundary condition and without any displacement. In fact, by considering this boundary condition and closing the deformations and shifts of the building facets in all directions, conditions are being prepared for examining merely the pattern of explosive loading on the building faces. Assuming that each variable has only 5 changes, and that these 5 times are included in all simulations, the number of simulations that must be done to simultaneously consider the four main variables is equal to $5^{4}=625$ because numerical simulations involve a large amount of time, using DOE industrial design methods to reduce the number of simulations while being comprehensive and efficient, and designing experiments in this research is done through the Taguchi method according to Table 4 [14]. If the angle of the building angle is more than 90 degrees, they are called open angles and are marked with a positive sign, and if the angle of the building angle is less than 90 degrees, they are called closed angles and are marked with a negative sign. In this study, the value of the zero-degree angle indicates that the vertical angle is corner angle.

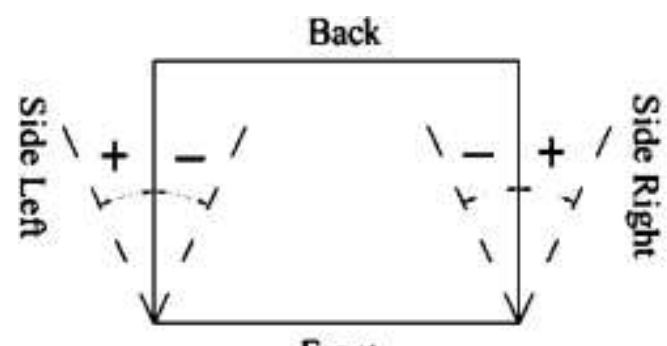

Front

Fig. 5. Change the angles of the building corner.

\section{Results and Discussion}

After simulating and analysing each experiment, the results of the AUTODYN software were obtained in the mean Time-Pressure diagram of each face is obtained from analytical method, and then the maximum pressure values $\left(\mathrm{P}_{\max }\right)$, the time of the explosion $\left(\mathrm{T}_{\mathrm{a}}\right)$, the corresponding time. Maximum pressure $\left(\mathrm{T}_{\mathrm{Pmax}}\right)$, positive phase completion time $\left(T_{o f}\right)$ and impulse value (area below the time pressure diagram in positive phase) were calculated for the side face and roof of the building. Depending on the changes in the amount of variables in each of the 25 simulations performed, each variable effects the loading components (Time-Pressure diagram) of each of the side face and roof of the building. In order to carefully investigated the effectiveness of each of the variables in the components of the load pattern (Time-Pressure diagram) of the building's side and roof, the results obtained from the software analysis of Design Expert software, which shows the effectiveness of each variable in each component of the Time-Pressure diagram as correlation coefficients in table 5 is provided.

\section{Table 5}

Coefficient of correlation of variables on the components of explosive loading of the side faces and roof of the building.

\begin{tabular}{|c|c|c|c|c|}
\hline & $\alpha_{\text {left }}$ & $\alpha_{\text {right }}$ & $R$ & $L$ \\
\hline$P_{\max }-R$ & -0.02 & -0.04 & -0.95 & -0.06 \\
\hline$T_{a}-R$ & 0.00 & -0.00 & 1.00 & 0.00 \\
\hline$T_{P_{\max }}-R$ & 0.00 & 0.00 & 1.00 & 0.00 \\
\hline$T_{o f}-R$ & -0.01 & -0.01 & 1.00 & -0.01 \\
\hline Impalse $-R$ & -0.23 & -0.11 & 0.72 & 0.38 \\
\hline$P_{\max }-S L$ & 0.59 & -0.07 & -0.50 & -0.30 \\
\hline$T_{a}-S L$ & 0.00 & -0.00 & 1.00 & 0.03 \\
\hline$T_{P_{\max }}-S L$ & -0.05 & 0.00 & 1.00 & 0.02 \\
\hline$T_{o f}-S L$ & -0.21 & 0.05 & 0.97 & -0.02 \\
\hline Impalse $-S L$ & 0.62 & -0.03 & 0.36 & -0.39 \\
\hline$P_{\max }-S R$ & -0.02 & 0.66 & -0.55 & -0.09 \\
\hline$T_{a}-S R$ & -0.01 & -0.00 & 1.00 & 0.02 \\
\hline$T_{P_{\max }}-S R$ & 0.00 & -0.06 & 0.99 & 0.03 \\
\hline$T_{o f}-S R$ & 0.04 & -0.21 & 0.97 & 0.01 \\
\hline Impalse $-S R$ & -0.01 & 0.67 & 0.47 & -0.22 \\
\hline & & & \multirow{2}{*}{\multicolumn{2}{|c|}{$\begin{array}{l}0.25 \sim 0.5 \\
0.75 \sim 1\end{array}$}} \\
\hline $0.5 \sim 0.75$ & & & & \\
\hline
\end{tabular}


Since the results obtained in Table 5 are two main conditions for reducing the number of simulations from 625 to 25 and simultaneously examining all variables in the simulations performed, for correlation coefficients less than 0.3 , the effective relationship between the two parameters cannot be investigated. In surface explosions, the blast wave front is initially hemispherical, which, as the blast wave progresses and increases in distance, becomes the Mach wave (smooth wave front) [3]. Considering the location of the building under study in this study compared to the location of the explosive, it is assumed that the building under investigation is within the Mach wave front. Therefore, the first collision of the Mach wave front is in front of the building, and the pressure applied to it in a moment and in all parts of its surface has a constant value. As the front of the building explodes and passes over the roof or sidewalls, at any point in time, only part of the roof surface and the surrounding walls are loaded, which puts a lot of pressure on the shock front, the position of the shock front. And the wavelength of the positive and negative phases of the explosion will depend [11]. In order to simplify the calculations, the mean TimePressure diagram, which includes a uniform pressure equal to the total roof surface and side faces, is considered. As can be seen in Table 5, the most important variable effecting the components of the time and side face pressure diagram of the building is the distance of the explosive from the building. As the distance of the explosive from the building increases, the maximum amount of pressure on the side face and roof of the building decreases. Also, as the distance of the explosive from the building increases, the time components of the mean pressure diagram of the side face and roof increase with a correlation coefficient of very close to 1 . In fact, following the increase in the distance of the explosive from the building, the rate of increase of time components increases compared to the decrease of pressure component in medium Time-Pressure curve of side faces and roof, and as a result, increases the area below this curve. The higher the correlation coefficient, the longer the positive phase of the diagram (direct effect of the explosive distance) than the lower the height of the diagram (the inverse effect of the explosive distance), increases the area under the Time-Pressure diagram (direct effect of the explosive distance on the impulse entering Building side faces). In fact, increasing the distance

ISSN (Print): 2256-6411 | ISSN (Online): 2456-6403 from the explosive to the building reduces the speed of the blast wave front and increases the impact time of the blast wave on the sides and roof of the building. Since the correlation coefficients related to the changes in the length of the front of the building are less than $40 \%$ on the components of explosive loading of side faces and roof and the progress of the blast wave is considered as Mach wave front (smooth wave front), it cannot be conclusively and accurately related these became the parameters. Therefore, based on the results obtained in Table 5, changes in the length of the front of the building will directly effect the duration of the blast wave on the front. First, the blast wave collides with the vertical line of the center of the front face, and then it spreads on the surface of the face, and over time it is emptied from the front face and transferred to the side faces and roof of the building. According to Table 5, increasing the length of the front side causes the maximum amount of pressure and the impulse to enter the side of the building to decrease, which indicates its inverse effect. Because changing the length of the front of the building affects the horizontal transmission of the wave on the surface of the front, it also has little effect on the maximum roof pressure of the building. But it has a direct and significant effect on the amount of roof impulse in the building. According to the conditions of this study, the simultaneous effects of the four variables are investigated the influencing factors on the roof loading pattern of the building are the wave front passing through the front along with the side of the building. In fact, changes in the angles of the building cause changes in the loading pattern of the sides, which, when the angle of the corner is opened outwards, make the side face almost similar to the front of the building in front of the blast wave front, and therefore cause The blast wave is transmitted from the side face to the roof surface of the building and affects the impulse entering the roof. The front of the blast wave forms a vortex after passing through the front face, and immediately after detaching from this surface. The pressure created on the side face and roof of the building is caused by the rolling of the waves on these surfaces [11]. If the angle of the corner of the building is right, the blast wave is transferred to the side face surfaces as shown in Figure 6 by forming a vortex in the corners of the building. Opening the left corner angle from the front face causes a smaller and smaller vortex to form, increasing the speed of the blast wave from the 
front face to the left side face. As the side face transfer speed increases, the time it takes for the wave to reach the surface of the side face decreases, and more pressure is transferred to the side face surface. The closure of the left corner angle from the front face is exactly the opposite. This means that as the angle of the left corner closes from the front face, a larger and larger vortex must be formed to move the blast wave front face from the front face to the left side face, which slows down the wave transfer speed to the left side face surface. If the velocity of the wave is reduced to the lateral side, the time of arrival of the wave on the surface of the lateral side will be increased and also less pressure will be transferred to the surface of the side face. As a result, changes in the angles of the building's corners affect the loading pattern of the side faces.

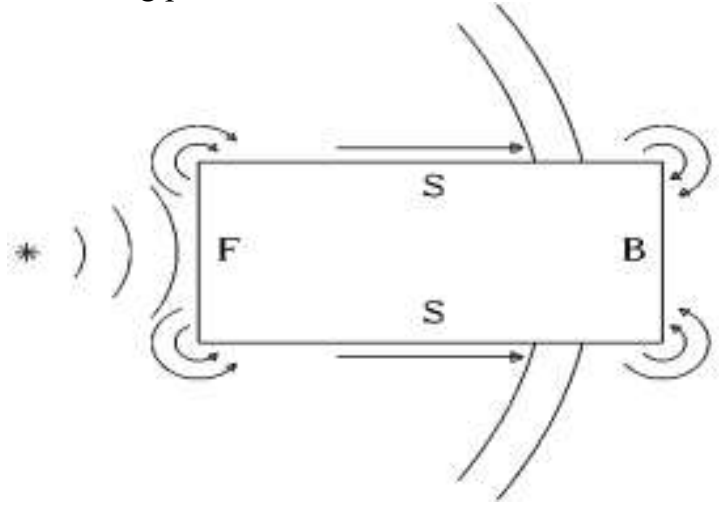

Fig. 6. Transfer the blast wave to the side faces and back of the building.

As the angle of the left corner opens from the front face of the building, the maximum amount of pressure and impulse on the left side face increases. Also, opening the angle can increase the wave velocity from the front face, and increase the velocity of the wave to the left. Changing the angles of the corner of the building changes the dimensions and shape of the roof of the building. As the angle of the building's angle increases, the velocity of the wave and the pressure of the side face increase and part of the force applied to the roof of the building is transmitted from the side faces, which increases the rate of displacement of the blast wave on the roof surface of the building and thus reduces the amount of roof impulse in the building. Therefore, changes in the angle of the left corner of the building from the front face have a direct effect on the maximum pressure value and impulse entering the left side face, and have the opposite effect on the amount of ISSN (Print): 2256-6411 | ISSN (Online): 2456-6403 impulse entering the roof of the building. The effect of the changes in the angle of the right corner of the building on the right side face and the roof of the building will be the same as the effect of the changes in the angle of the left corner. In order to observe the separate effect of each of the variables on the components of loading the side faces and roof of the building, the following figures are presented based on their correlation coefficients.

\section{Comparing the side faces loading pattern with the UFC loading diagram}

In a number of simulations performed in this study, the angles of the building's corner are wider than the front face of the building and form a more open angle. As the angle of the building angle opens, the position of the corresponding face relative to the location of the explosive becomes almost the same as the position of the front face of the building. In the UFC regulations, relationships and diagrams are presented in order to calculate the proposed TimePressure diagram of the front face of the building in the case that it has an angle to the location of the explosive. In this study, for cases where the open angles and position of the corresponding side angle are close to the front face of the building, the TimePressure diagram of that side face can be obtained using the UFC code as well as the front side of the building. As a result, in order to analyse the sensitivity of this issue in the simulations performed in this study, the proposed time pressure graphs of UFC regulations compared to the medium time pressure diagrams obtained from numerical simulation in AUTODYN software are compared and investigated. In order to investigate the nonlinear time pressure diagram obtained from numerical simulation for the front face of the building, it is better to modify it as a linear UFC time pressure diagram linearly. And its impulse value (area below the diagram in the positive phase) is a modified linear time pressure diagram for the front face of the building. According to Figure 11, the value of $t_{a}$ is the time the wave reaches the foot of the structure. The time it takes for the wave to reach the foot of the structure until it reaches the maximum pressure value seen in most of the simulation diagrams is $t_{r}$. The maximum pressure value is indicated by $\mathrm{P}_{\mathrm{n}}$ and $\mathrm{P}_{\mathrm{m}}$ indicates the new maximum pressure. In this study, relying on the principle of constant value of impulse and correction of the time 
pressure triangle diagram to the vertical angle triangle, the new maximum pressure value is obtained using the following relations:

$$
\begin{aligned}
t_{o f} & =\frac{2 I_{n}}{P_{n}} \\
P_{m} & =\frac{2 I_{n}}{t_{o f}^{\prime}} \\
t_{o f}^{\prime} & =t_{o f}-t_{r}
\end{aligned}
$$

Since the side angle corresponding to the open angle is considered to be the same as the front face of the building, the time pressure diagram obtained from the numerical simulation is examined in both nonlinear and linear modified modes.

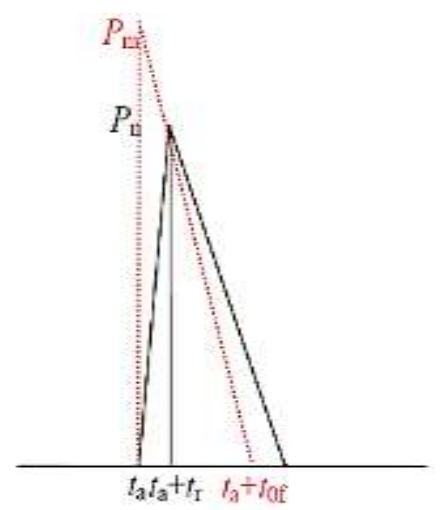

Fig. 7. Design of numerical modification method.

In Table 4, this study needs to be performed only for experiments whose angles have changed with a positive sign. Therefore, comparing time pressure charts for Tests $4,5,9,10,14,15$ to 25 needs to be done. Since the number of diagrams in this section is too large for all simulations and only a comparison between them is the criterion, compare the time pressure chart obtained from the UFC code with the AUTODYN Time-Pressure diagram in both nonlinear and linear modified modes on the right and left side face of the simulation the number (25) is shown in Figure 12 as an example. Table 6 shows the values of the main components of each of these specified simulations, as well as the percentage of error between the proposed Time-Pressure diagram of the UFC code and the average Time-Pressure diagram obtained from the numerical simulation.

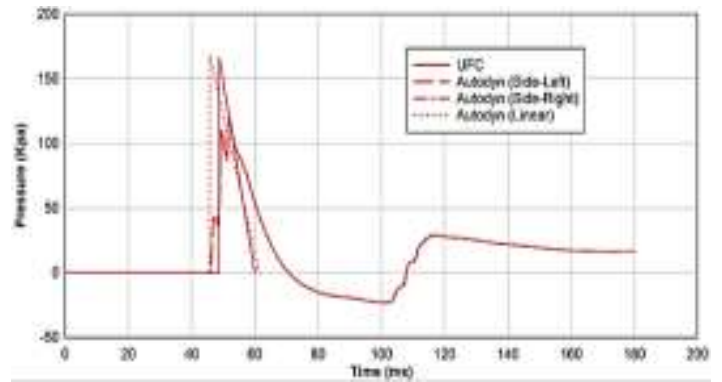

Fig. 8. Comparison of UFC Time-Pressure diagram with AUTODYN.

In Table 6, where in each simulation the percentage of error between the time components has small values, but the error of the main components of the maximum pressure and the impulse entering the face has a high percentage. In this study and the results obtained, for the aspect that the angle of the corresponding angle is opened relative to the front of the building (the position of the side face position relative to the location of the explosive is almost close to the position of the front side of the building and the side face as The front side is considered), the pressure diagram of the proposed time UFC code cannot be matched with the numerical simulation results.

\section{Conclusions}

There are different methods and solutions for analysing and exploring an explosive phenomenon. One way to investigate explosive phenomena is to use the UFC-3-340-02 regulations. The use of UFC regulations must be within the scope of a number of simplifying assumptions, one of which can no longer be relied upon to ensure the results of the UFC regulations. Therefore, if the characteristics of an explosive phenomenon are not within the scope of the simplistic conditions and assumptions of the UFC Code, numerical or experimental methods should be used to calculate and obtain appropriate results. In this study, the effects of violating the correctness of the right angles of the building corner, which is one of the simplifying assumptions of the UFC code, have been investigated. In this study, the effects of the four variables of the left corner angle from the front face $\left(\alpha_{\text {left }}\right)$, the right corner angle from the front face $\left(\alpha_{\text {right }}\right)$, the distance of the explosive to the building (R) and the length of the front face (L) on the main components of explosive loading side faces and the roof of the building, including the 
maximum pressure $\left(P_{\max }\right)$, the time of arrival of the blast wave $\left(T_{a}\right)$, the time corresponding to the maximum pressure $\left(T_{P \max }\right)$, the time of completion of the positive phase $\left(T_{o f}\right)$ and the amount of impulse (I) were examined. According to the studies conducted in this study, the following results were obtained:

1. The distance of the explosive to the building inversely affects the velocity of the blast wave front and the maximum pressure on the side face and roof of the building and also directly effects the time of the blast and the duration of the wave on the building. The main cause of impulse change is the input to the side face and roof the maximum pressure to the input. According to Table 5 and Figure 9, the opposite has happened, which seems to be due to the fact that the increase in impulse rate is due to the fact that the rate of increase in time components has increased compared to the decrease in compression component and has a direct effect on impulse.

2. As the angle of each side faces of the building becomes more open with the front face, the maximum pressure and the impulse entering that side face increase. Also, opening the angle can increase the speed of wave transfer from the front face to the side face. Changing the angles of the building corner also changes the dimensions and shape of the roof of the building, which can effect the loading components of the roof of the building. The angle changes of each side of the side with the front face of the building have a direct effect on the maximum pressure and the impulse entering that side face.

3. In investigating the effect of building corner angles on the explosive loading components of each aspect, which with the opening of building corner angles (angles greater than $90^{\circ}$ ), the speed of the blast wave on the side faces increases and the blast wave current covers the building at a higher speed. As the speed of the wave passes through the building, the duration of the wave's effect on the building will decrease. Therefore, the geometry of the building plan has a significant effect on the loading of the explosion on its surface. By selecting the cross section of the building with angles greater than $90^{\circ}$, the blast wave front will pass through the building at a higher speed, but the maximum pressure entering the side faces will increase. Since the position of an explosive phenomenon is unpredictable compared to a building, the design of the building, which has the same resistance to the blast wave and passes the blast wave at a higher speed, can have an ideal resistance to the blast wave front. The closer the geometry of the plan of the building in the form of a circle, the faster the blast wave will pass through the building and will cause less damage.

\section{References}

[1] A. Jafari, Q. Sadrnejad, A. s. Darian, h. A. B. Pour, Investigation of the Explosion Effect on RC Structures, Journal of Inactive Defense, Vol. 13, No. 2, pp. 6755,2010

[2] N. Gebbeken, T. Döge, Explosion protection architectural design, urban planning and landscape planning, International Journal of Protective Structures, Vol. 1, No. 1, pp. 1-21, 2010.

[3] U. DoD, Structures to resist the effects of accidental explosions, Unified Facilities Criteria, United States of America, Department of Defense, Washington, DC, Document No. UFC, pp. 3-340, 2008.

[4] S. E. Rigby, S. D. Fay, A. Tyas, J. A. Warren, S. D. Clarke, Angle of incidence effects on far-field positive and negative phase blast parameters, International Journal of Protective Structures, Vol. 6, No. 1, pp. 2342, 2015.

[5] Wang G, Lu W, Yang G, Yan P, Chen M, Zhao X, Li Q. A state-of-the-art review on blast resistance and protection of high dams to blast loads. International Journal of Impact Engineering. 2020 Feb 4:103529.

[6] Yang G, Huang W, Feng S. Anti-explosion Performance of Engineered Cementitious Composite Explosion-Proof Wall. Advances in Materials Science and Engineering. 2020;2020.

[7] Rashad M, Wahab M, Yang TY. Experimental and numerical investigation of RC sandwich panels with helical springs under free air blast loads. Steel and Composite Structures. 2019;30(3):217-30.

[8] Metzger BD, Margalit B, Sironi L. Fast radio bursts as synchrotron maser emission from decelerating relativistic blast waves. Monthly Notices of the Royal Astronomical Society. 2019 May;485(3):4091-106.

[9] S. Zahmatkesh, Investigation of Charges Due to Expendable Contact Explosion with Conventional Geometric Shapes, Thesis, Imam Hussein University of Technology, Non-Agent Defense School and Research, 2016.

[10] M. Bangash, Impact and explosion: structural analysis and design: Blackwell Scientific Publications, 1992. 
[11] S. Glasstone, P. J. Dolan, The effects of nuclear weapons, department of defesnse Washington dc, pp. 1977.

[12] S. S. Amiruddin, M. Ziaei, Investigation of the Effect of Explosion on the Function of Beamto-Spindle Column Joints, Journal of Passive Defense, Vol. 14, No. 1, pp. 57 45, 2012.

[13] T. PDC, 06-01," Methodology Manual for the Single-Degree-of-Freedom Blast Effects Design Spreadsheets (SBEDS), US Army Corps of Engineers Protective Design Center, 2006.

[14] M Abual-Bashri, c. A. Aurora, Introduction to Optimal Design, pp. 855: Ferdowsi University of Mashhad, 1999.

[15] Ling Y, Balachandar S. Simulation and scaling analysis of a spherical particle-laden blast wave. Shock Waves. 2018 May 1;28(3):54558.
[16]Kim, Tae Jin, and Yoon-Suk Chang. "Investigation of blast wave effects on containment wall and steam generator." ASME 2018 Pressure Vessels and Piping Conference. American Society of Mechanical Engineers Digital Collection, 2018.

[17]Ivančo, Matúš, Lucia Figuli, and Chiara Bedon. "Different Approaches of Numerical Simulation of Blast for Civil Engineering Applications." Soft Target Protection. Springer, Dordrecht, 2020. 169-181.

[18] Holgado, David \& Waclawczyk, Johnny \& Collins worth, Jerry \& Prakash, Pradeep. New Building Design Considerations for Petrochemical Facilities, 2014.

[19] Stochino, Flavio. Exceptional Actions: Blast Loads on Reinforced Concrete Structures, 2013.

Table 2

Comparison of the results of Time-Pressure diagrams of the side faces and roof of the building.

\begin{tabular}{|c|c|c|c|c|}
\hline & $\begin{array}{l}p_{\max } \\
(K p a)\end{array}$ & $\begin{array}{c}t_{a} \\
(m s)\end{array}$ & $\begin{array}{c}t_{o f} \\
(m s)\end{array}$ & $i$ \\
\hline UFC & 110.87 & 17.98 & 37.7 & 1093.39 \\
\hline Side Left (Analytical) & 122.7 & 19.82 & 38.82 & 1048.17 \\
\hline Side Right (Analytical) & 123.27 & 19.90 & 38.91 & 1048.31 \\
\hline Roof (Analytical) & 126.01 & 19.82 & 38.82 & 1023.69 \\
\hline Side Left (Numerical) & 122.35 & 19.82 & 38.82 & 1043.31 \\
\hline Side Right (Numerical) & 122.93 & 19.90 & 38.92 & 1043.37 \\
\hline Roof (Numerical) & 125.15 & 19.82 & 38.82 & 1018.64 \\
\hline Percentage of error between Side Left (Analytical) and UFC & $10.67 \%$ & $10.23 \%$ & $2.97 \%$ & $4.14 \%$ \\
\hline Percentage of error between UFC and Side Right (Analytical) & $11.18 \%$ & $10.68 \%$ & $3.21 \%$ & $4.13 \%$ \\
\hline Percentage of error between UFC and Roof (Analytical) & $13.66 \%$ & $10.23 \%$ & $2.97 \%$ & $6.37 \%$ \\
\hline Percentage of error between UFC and Side Left (Numerical) & $10.35 \%$ & $10.23 \%$ & $2.97 \%$ & $4.58 \%$ \\
\hline Percentage of error between UFC and Side Right (Numerical) & $10.88 \%$ & $10.68 \%$ & $3.24 \%$ & $4.57 \%$ \\
\hline Percentage of error between UFC and Roof (Numerical) & $12.88 \%$ & $10.23 \%$ & $2.97 \%$ & $6.84 \%$ \\
\hline Percentage of error between Side Left (Analytical) and Side Left (Numerical) & $0.29 \%$ & $0.00 \%$ & $0.00 \%$ & $0.47 \%$ \\
\hline Percentage of error between Side Right (Analytical) and Side Right (Numerical) & $0.28 \%$ & $0.00 \%$ & $0.03 \%$ & $0.47 \%$ \\
\hline Percentage of error between Roof (Analytical) and Roof (Numerical) & $0.69 \%$ & $0.00 \%$ & $0.00 \%$ & $0.49 \%$ \\
\hline
\end{tabular}

Table 3

Numerical ranges of variables effecting the explosive loading pattern.

\begin{tabular}{|l|c|c|c|c|c|}
\hline \multicolumn{1}{|c|}{ Variable } & $\begin{array}{c}\text { The first } \\
\text { factor }\end{array}$ & $\begin{array}{c}\text { The second } \\
\text { factor }\end{array}$ & $\begin{array}{c}\text { The third } \\
\text { factor }\end{array}$ & $\begin{array}{c}\text { The fourth } \\
\text { factor }\end{array}$ & $\begin{array}{c}\text { The fifth } \\
\text { factor }\end{array}$ \\
\hline The left corner angle of the front face $\left(\alpha_{\text {left }}\right)$ & -45 & -22.5 & 0 & 22.5 & 45 \\
\hline The right corner angle of the front face $\left(\alpha_{\text {right }}\right)$ & -45 & -22.5 & 0 & 22.5 & 45 \\
\hline Front face length $(\mathrm{L})$ & $6 \mathrm{~m}$ & $7.5 \mathrm{~m}$ & $9 \mathrm{~m}$ & $10.5 \mathrm{~m}$ & $12 \mathrm{~m}$ \\
\hline The distance of the explosive material from the building $(\mathrm{R})$ & $10 \mathrm{~m}$ & $17.5 \mathrm{~m}$ & $25 \mathrm{~m}$ & $32.5 \mathrm{~m}$ & $40 \mathrm{~m}$ \\
\hline
\end{tabular}


Table 4

Design of numerical simulations by Taguchi method.

\begin{tabular}{|c|c|c|c|c|c|c|c|c|c|c|c|c|c|}
\hline $\mathrm{n}$. & 1 & 2 & 3 & 4 & 5 & 6 & 7 & 8 & 9 & 10 & 11 & 12 & 13 \\
\hline$\alpha_{\text {left }}$ & -45 & -45 & -45 & -45 & -45 & -22.5 & -22.5 & -22.5 & -22.5 & -22.5 & 0 & 0 & 0 \\
\hline$\alpha_{\text {right }}$ & -45 & -22.5 & 0 & 22.5 & 45 & -45 & -22.5 & 0 & 22.5 & 45 & -45 & -22.5 & 0 \\
\hline$R$ & 10 & 17.5 & 25 & 32.5 & 40 & 17.5 & 25 & 32.5 & 40 & 10 & 25 & 32.5 & 40 \\
\hline$L$ & 12 & 10.5 & 9 & 7.5 & 6 & 9 & 7.5 & 6 & 12 & 10.5 & 6 & 12 & 10.5 \\
\hline \hline $\mathrm{n}$. & 14 & 15 & 16 & 17 & 18 & 19 & 20 & 21 & 22 & 23 & 24 & 25 & \\
\hline$\alpha_{\text {left }}$ & 0 & 0 & 22.5 & 22.5 & 22.5 & 22.5 & 22.5 & 45 & 45 & 45 & 45 & 45 & \\
\hline$\alpha_{\text {right }}$ & 22.5 & 45 & -45 & -22.5 & 0 & 22.5 & 45 & -45 & -22.5 & 0 & 22.5 & 45 & - \\
\hline$R$ & 10 & 17.5 & 32.5 & 40 & 10 & 17.5 & 25 & 40 & 10 & 17.5 & 25 & 32.5 \\
\hline$L$ & 9 & 7.5 & 10.5 & 9 & 7.5 & 6 & 12 & 7.5 & 6 & 12 & 10.5 & 9 & \\
\hline
\end{tabular}

\section{Left Angel}

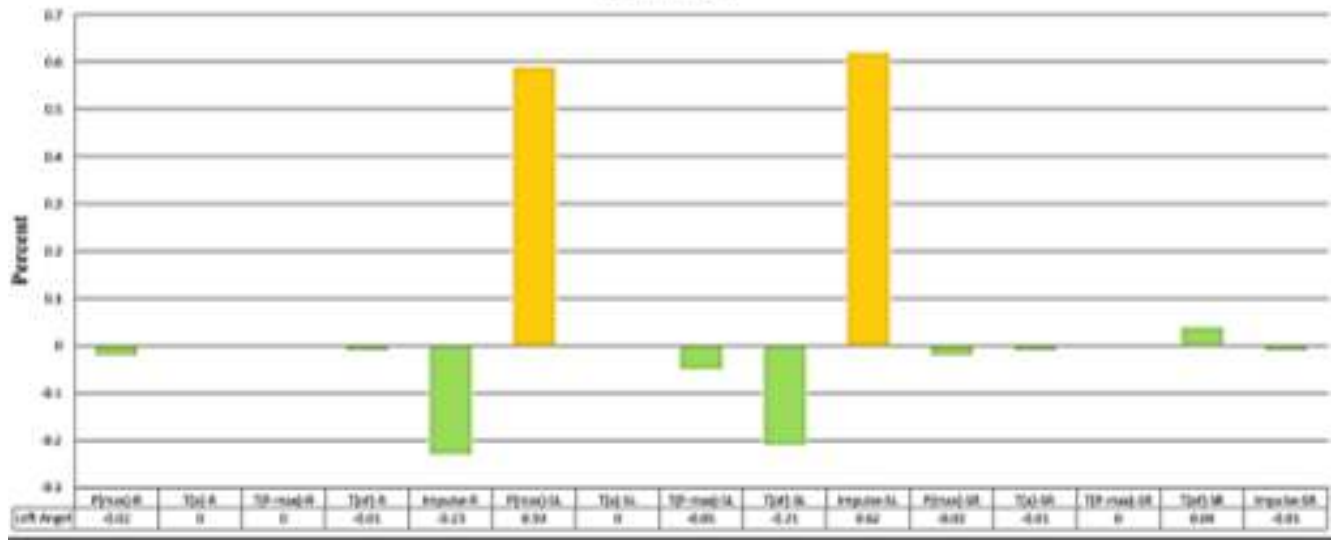

Fig. 9. The effect of $\alpha_{\text {Left }}$ variable on the explosive loading components of the side faces and roof of the building.

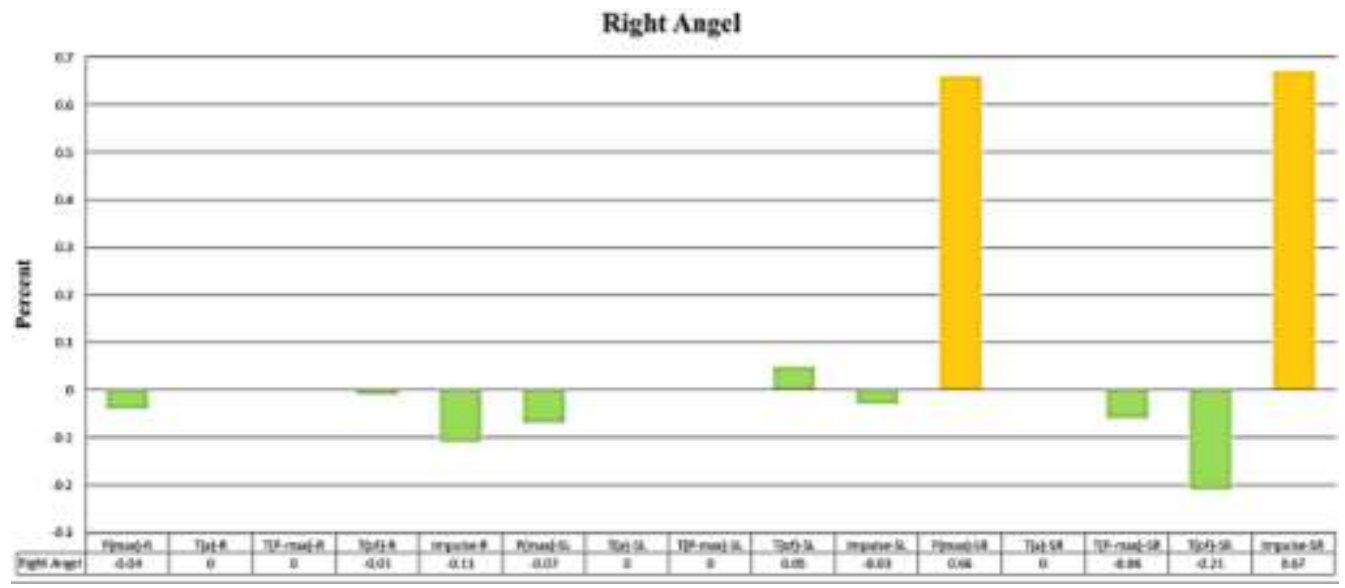

Fig. 10. The effect of $\alpha_{\text {Right }}$ variable on the explosive loading components of the side faces and roof of the building. 


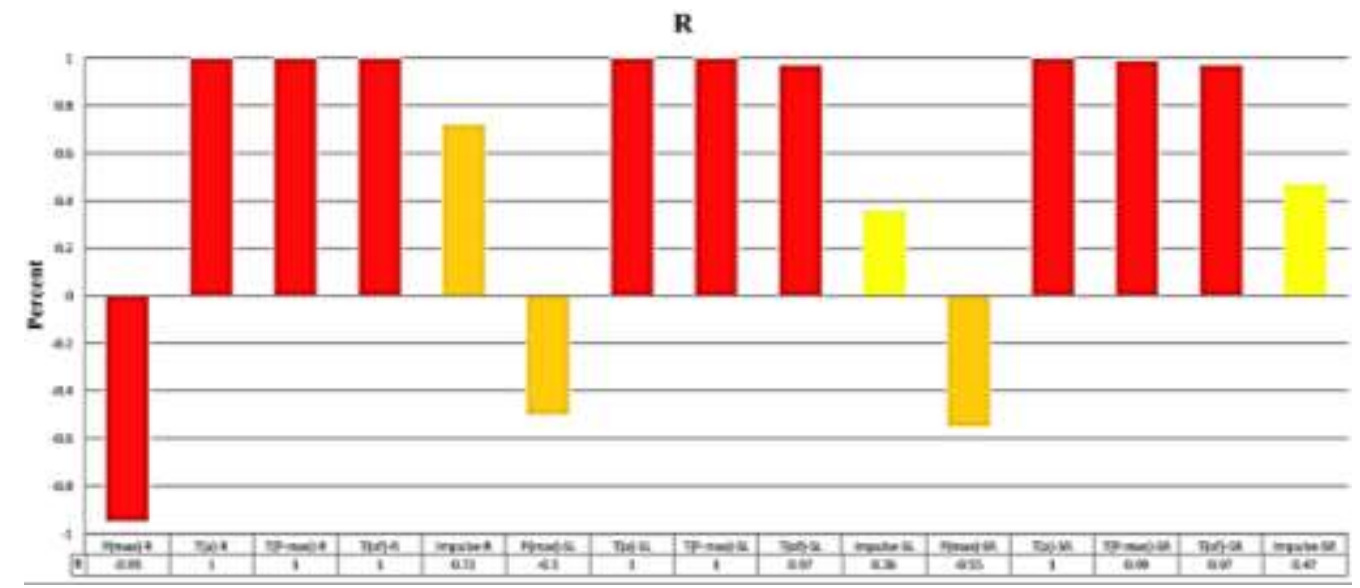

Fig. 11. Effect of $R$ variable on the explosive loading components of the side faces and roof of the building.

L

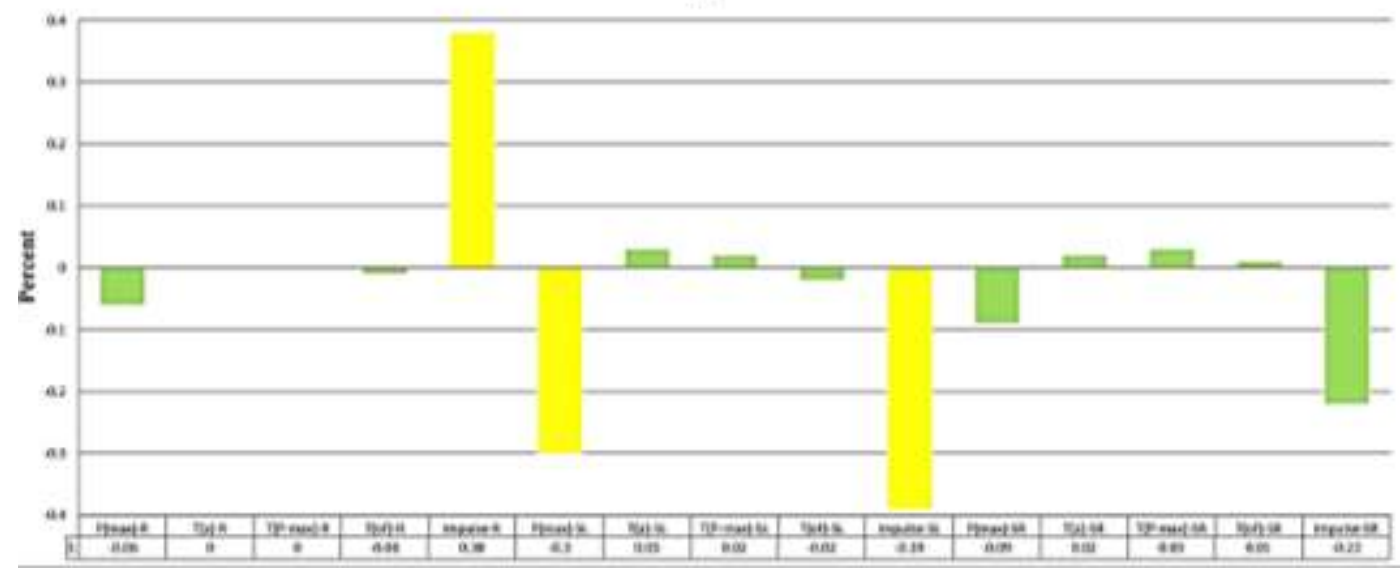

Fig. 12. The effect of variable $L$ on the explosive loading components of the side faces and roof of the building.

Table 6

Comparison of parameters related to time pressure charts obtained from UFC regulations and numerical simulation in AUTODYN software in two modified nonlinear and linear modes.

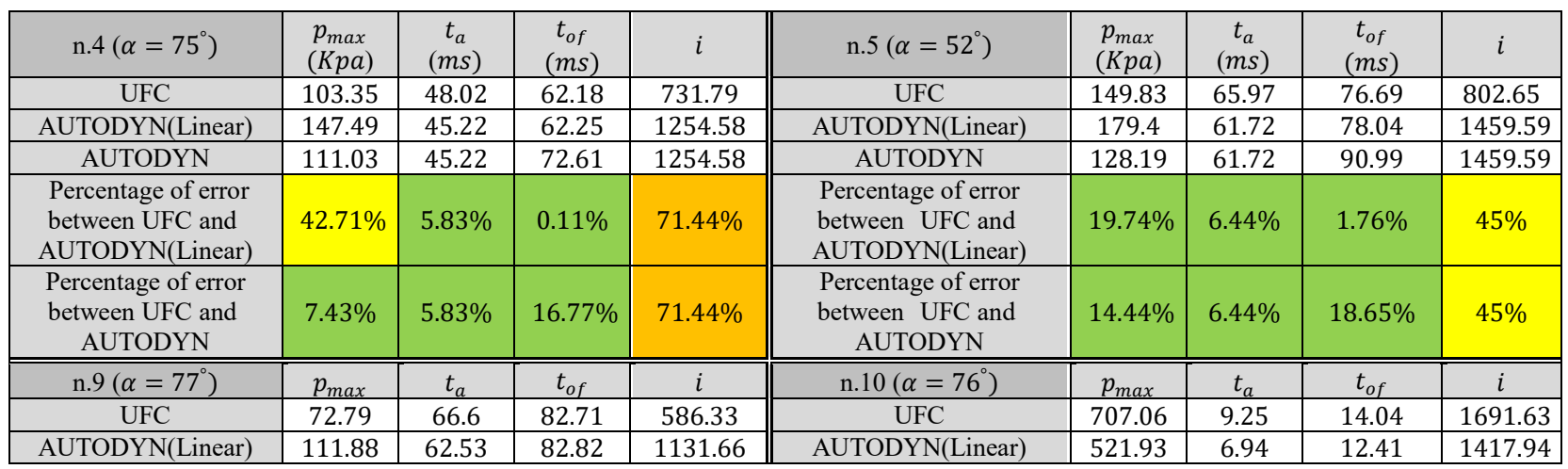




\begin{tabular}{|c|c|c|c|c|c|c|c|c|c|}
\hline AUTODYN & 86.09 & 62.53 & 98.6 & 1131.66 & AUTODYN & 368.85 & 6.94 & 17.52 & 1417.94 \\
\hline $\begin{array}{l}\text { Percentage of error } \\
\text { between UFC and } \\
\text { AUTODYN(Linear) }\end{array}$ & $53.7 \%$ & $6.11 \%$ & $0.13 \%$ & $93 \%$ & $\begin{array}{l}\text { Percentage of error } \\
\text { between UFC and } \\
\text { AUTODYN(Linear) }\end{array}$ & $26.18 \%$ & $24.97 \%$ & $11.61 \%$ & $16.18 \%$ \\
\hline $\begin{array}{l}\text { Percentage of error } \\
\text { between UFC and } \\
\text { AUTODYN } \\
\end{array}$ & $18.27 \%$ & $6.11 \%$ & $19.21 \%$ & $93 \%$ & $\begin{array}{l}\text { Percentage of error } \\
\text { between UFC and } \\
\text { AUTODYN } \\
\end{array}$ & $47.83 \%$ & $24.97 \%$ & $24.79 \%$ & $16.18 \%$ \\
\hline n.14 $\left(\alpha=41^{\circ}\right)$ & $p_{\max }$ & $t_{a}$ & $t_{o f}$ & $i$ & $\mathrm{n} .15\left(\alpha=61^{\circ}\right)$ & $p_{\max }$ & $t_{a}$ & $t_{o f}$ & $i$ \\
\hline UFC & 639.19 & 8.18 & 12.56 & 1399.43 & UFC & 371.09 & 18.53 & 26.49 & 1475.92 \\
\hline AUTODYN(Linear) & 364.8 & 6.49 & 12.00 & 996.33 & AUTODYN(Linear) & 290.39 & 16.02 & 25.93 & 1437.91 \\
\hline AUTODYN & 272.14 & 6.49 & 16.22 & 996.33 & AUTODYN & 223.41 & 16.02 & 31.31 & 1437.91 \\
\hline $\begin{array}{l}\text { Percentage of error } \\
\text { between UFC and } \\
\text { AUTODYN(Linear) }\end{array}$ & $42.93 \%$ & $20.66 \%$ & $4.46 \%$ & $28.8 \%$ & $\begin{array}{l}\text { Percentage of error } \\
\text { between UFC and } \\
\text { AUTODYN(Linear) }\end{array}$ & $21.75 \%$ & $13.55 \%$ & $2.11 \%$ & $2.58 \%$ \\
\hline $\begin{array}{c}\text { Percentage of error } \\
\text { between UFCand } \\
\text { AUTODYN } \\
\end{array}$ & $57.42 \%$ & $20.66 \%$ & $29.14 \%$ & $28.8 \%$ & $\begin{array}{c}\text { Percentage of error } \\
\text { between UFC and } \\
\text { AUTODYN } \\
\end{array}$ & $39.79 \%$ & $13.55 \%$ & $18.19 \%$ & $2.58 \%$ \\
\hline $\mathrm{n} .16\left(\alpha=77^{\circ}\right)$ & $p_{\max }$ & $t_{a}$ & $t_{o f}$ & $i$ & $\mathrm{n} .17\left(\alpha=75^{\circ}\right)$ & $p_{\max }$ & $t_{a}$ & $t_{o f}$ & $i$ \\
\hline UFC & 98.68 & 48.55 & 62.87 & 706.47 & UFC & 77.24 & 66.07 & 81.78 & 606.64 \\
\hline AUTODYN(Linear) & 128.94 & 45.68 & 63.58 & 1154.19 & AUTODYN(Linear) & 120.78 & 61.91 & 82.14 & 1221.96 \\
\hline AUTODYN & 96.99 & 45.68 & 74.99 & 1154.19 & AUTODYN & 94.24 & 61.91 & 96.34 & 1221.96 \\
\hline $\begin{array}{l}\text { Percentage of error } \\
\text { between UFC and } \\
\text { AUTODYN(Linear) }\end{array}$ & $30.66 \%$ & $5.91 \%$ & $1.13 \%$ & $63.37 \%$ & $\begin{array}{l}\text { Percentage of error } \\
\text { between UFC and } \\
\text { AUTODYN(Linear) }\end{array}$ & $56.37 \%$ & $6.29 \%$ & $0.44 \%$ & $99.43 \%$ \\
\hline $\begin{array}{c}\text { Percentage of error } \\
\text { between UFC and } \\
\text { AUTODYN } \\
\end{array}$ & $1.71 \%$ & $5.91 \%$ & $19.28 \%$ & $63.37 \%$ & $\begin{array}{c}\text { Percentage of error } \\
\text { between UFC and } \\
\text { AUTODYN } \\
\end{array}$ & $22 \%$ & $6.29 \%$ & $17.8 \%$ & $99.43 \%$ \\
\hline $\mathrm{n} .18\left(\alpha=88^{\circ}\right)$ & $p_{\max }$ & $t_{a}$ & $t_{o f}$ & $i$ & n.20(S-L) $\left(\alpha=82^{\circ}\right)$ & $p_{\max }$ & $t_{a}$ & $t_{o f}$ & $i$ \\
\hline UFC & 698.98 & 7.85 & 12.11 & 1486.26 & UFC & 138.14 & 32.75 & 44.22 & 792.56 \\
\hline AUTODYN(Linear) & 397.82 & 6.18 & 11.76 & 1110.69 & AUTODYN(Linear) & 146.71 & 30.63 & 45.96 & 1127.08 \\
\hline AUTODYN & 300.97 & 6.18 & 15.91 & 1110.69 & AUTODYN & 110 & 30.63 & 52.57 & 1127.08 \\
\hline $\begin{array}{l}\text { Percentage of error } \\
\text { between UFC and } \\
\text { AUTODYN(Linear) }\end{array}$ & $43.09 \%$ & $21.27 \%$ & $2.89 \%$ & $25.27 \%$ & $\begin{array}{l}\text { Percentage of error } \\
\text { between UFC and } \\
\text { AUTODYN(Linear) }\end{array}$ & $6.2 \%$ & $6.47 \%$ & $3.93 \%$ & $42.21 \%$ \\
\hline $\begin{array}{l}\text { Percentage of error } \\
\text { between UFC and } \\
\text { AUTODYN } \\
\end{array}$ & $56.94 \%$ & $21.27 \%$ & $2.89 \%$ & $25.27 \%$ & $\begin{array}{c}\text { Percentage of error } \\
\text { between UFC and } \\
\text { AUTODYN } \\
\end{array}$ & $20.37 \%$ & $6.47 \%$ & $18.88 \%$ & $42.21 \%$ \\
\hline n. $20(\mathrm{~S}-\mathrm{R})\left(\alpha=62^{\circ}\right)$ & $p_{\max }$ & $t_{a}$ & $t_{o f}$ & $i$ & n.21 $\left(\alpha=53^{\circ}\right)$ & $p_{\max }$ & $t_{a}$ & $t_{o f}$ & $i$ \\
\hline UFC & 202.39 & 33.39 & 43.89 & 1063.14 & UFC & 148.21 & 66.21 & 76.91 & 792.86 \\
\hline AUTODYN(Linear) & 216.49 & 30.5 & 42.84 & 1330.29 & AUTODYN(Linear) & 159.58 & 61.91 & 79.09 & 1363.43 \\
\hline AUTODYN & 143.99 & 30.5 & 52.9 & 1330.29 & AUTODYN & 114.53 & 61.91 & 91.99 & 1363.43 \\
\hline $\begin{array}{l}\text { Percentage of error } \\
\text { between UFC and } \\
\text { AUTODYN(Linear) }\end{array}$ & $6.97 \%$ & $8.66 \%$ & $2.39 \%$ & $25.13 \%$ & $\begin{array}{l}\text { Percentage of error } \\
\text { between UFC and } \\
\text { AUTODYN(Linear) }\end{array}$ & $7.67 \%$ & $6.49 \%$ & $2.83 \%$ & $71.96 \%$ \\
\hline $\begin{array}{l}\text { Percentage of error } \\
\text { between UFC and } \\
\text { AUTODYN }\end{array}$ & $28.86 \%$ & $8.66 \%$ & $20.53 \%$ & $25.13 \%$ & $\begin{array}{c}\text { Percentage of error } \\
\text { between UFC and } \\
\text { AUTODYN } \\
\end{array}$ & $22.72 \%$ & $6.49 \%$ & $19.61 \%$ & $71.96 \%$ \\
\hline $\mathrm{n} .22\left(\alpha=68^{\circ}\right)$ & $p_{\max }$ & $t_{a}$ & $t_{o f}$ & $i$ & $\mathrm{n} .23\left(\alpha=67^{\circ}\right)$ & $p_{\max }$ & $t_{a}$ & $t_{o f}$ & $i$ \\
\hline UFC & 1094.63 & 8.03 & 11.95 & 2142.35 & UFC & 296.52 & 19.81 & 28.55 & 1296.38 \\
\hline AUTODYN(Linear) & 706.2 & 6.07 & 11.22 & 1815.99 & AUTODYN(Linear) & 238.76 & 17.34 & 28.28 & 1306.62 \\
\hline AUTODYN & 515.32 & 6.07 & 15.88 & 1815.99 & AUTODYN & 186.83 & 17.34 & 33.47 & 1306.62 \\
\hline $\begin{array}{l}\text { Percentage of error } \\
\text { between UFC and } \\
\text { AUTODYN(Linear) }\end{array}$ & $35.49 \%$ & $24.41 \%$ & $6.11 \%$ & $15.23 \%$ & $\begin{array}{l}\text { Percentage of error } \\
\text { between UFC and } \\
\text { AUTODYN(Linear) }\end{array}$ & $19.48 \%$ & $12.47 \%$ & $0.95 \%$ & $0.79 \%$ \\
\hline $\begin{array}{l}\text { Percentage of error } \\
\text { between UFC and } \\
\text { AUTODYN } \\
\end{array}$ & $52.92 \%$ & $24.41 \%$ & $32.89 \%$ & $15.23 \%$ & $\begin{array}{l}\text { Percentage of error } \\
\text { between UFC and } \\
\text { AUTODYN } \\
\end{array}$ & $36.99 \%$ & $12.47 \%$ & $17.23 \%$ & $0.79 \%$ \\
\hline n.24(S-L) $\left(\alpha=60^{\circ}\right)$ & $p_{\max }$ & $t_{a}$ & $t_{o f}$ & $i$ & n.24 (S-R) $\left(\alpha=80^{\circ}\right)$ & $p_{\max }$ & $t_{a}$ & $t_{o f}$ & $i$ \\
\hline
\end{tabular}




\begin{tabular}{|c|c|c|c|c|c|c|c|c|c|}
\hline UFC & 215.69 & 32.97 & 43.17 & 1100.36 & UFC & 144.15 & 32.39 & 43.82 & 824.22 \\
\hline $\begin{array}{l}\text { Percentage of error } \\
\text { between UFC and } \\
\text { AUTODYN(Linear) }\end{array}$ & $4.46 \%$ & $7.83 \%$ & $1.89 \%$ & $22.3 \%$ & $\begin{array}{l}\text { Percentage of error } \\
\text { between UFC and } \\
\text { AUTODYN(Linear) }\end{array}$ & $8.29 \%$ & $6.79 \%$ & $4.27 \%$ & $46.54 \%$ \\
\hline $\begin{array}{c}\text { Percentage of error } \\
\text { between UFC and } \\
\text { AUTODYN } \\
\end{array}$ & $29.88 \%$ & $7.83 \%$ & $21.06 \%$ & $22.3 \%$ & $\begin{array}{l}\text { Percentage of error } \\
\text { between UFC and } \\
\text { AUTODYN }\end{array}$ & $19.11 \%$ & $6.79 \%$ & $17.46 \%$ & $46.54 \%$ \\
\hline UFC & 283.37 & 17.77 & 25.7 & 1124.29 & UFC & 166.09 & 48.73 & 59.97 & 934.02 \\
\hline AUTODYN(Linear) & 240.66 & 15.59 & 25.25 & 1159.12 & AUTODYN(Linear) & 168.31 & 45.64 & 61.48 & 1330.39 \\
\hline AUTODYN (S-Left) & 167.05 & 15.59 & 31.85 & 1159.21 & AUTODYN (S-Left) & 120.98 & 45.64 & 70.64 & 1330.39 \\
\hline AUTODYN (S-Right) & 167.03 & 15.59 & 31.85 & 1159.12 & AUTODYN (S-Right) & 119.28 & 45.64 & 70.64 & 1339.83 \\
\hline $\begin{array}{l}\text { Percentage of error } \\
\text { between UFC and } \\
\text { AUTODYN(Linear) }\end{array}$ & $15.07 \%$ & $12.27 \%$ & $1.75 \%$ & $3.09 \%$ & $\begin{array}{l}\text { Percentage of error } \\
\text { between UFC and } \\
\text { AUTODYN(Linear) }\end{array}$ & $1.34 \%$ & $6.34 \%$ & $2.52 \%$ & $42.44 \%$ \\
\hline $\begin{array}{l}\text { Percentage of error } \\
\text { between UFC and } \\
\text { AUTODYN (S-Right) }\end{array}$ & $41.05 \%$ & $12.27 \%$ & $23.93 \%$ & $3.09 \%$ & $\begin{array}{l}\text { Percentage of error } \\
\text { between UFC and } \\
\text { AUTODYN (S-Right) }\end{array}$ & $28.18 \%$ & $6.34 \%$ & $17.79 \%$ & $43.44 \%$ \\
\hline
\end{tabular}

